\title{
Butafosfan e cianocobalamina: estratégia para melhorar a funcionalidade hepática de vacas leiteiras primíparas*
}

\section{Butaphosphan and cyanocobalamin: strategy to improve the liver function of primiparous dairy cows}

\author{
Marcelo Moreira Antunes, ${ }^{* *}$ Rubens Alves Pereira, ${ }^{* *}$ Paula Montagner, ${ }^{* *}$ Rafael da Fonseca Prietsch, ${ }^{* *}$ \\ Josiane de Oliveira Feijo, ${ }^{* *}$ Camila Pizoni, ${ }^{* *}$ Francisco Augusto Burkert Del Pino, ${ }^{* *}$ Marcio Nunes Corrêa ${ }^{* *}$
}

\begin{abstract}
Resumo
O objetivo deste estudo foi avaliar os efeitos do butafosfan isolado ou associado à cianocobalamina sobre marcadores do metabolismo energético, mineral e inflamatório, produção de leite e índice de funcionalidade hepática (IFH) de vacas leiteiras primíparas. Vinte e três vacas da raça Holandês foram aleatoriamente distribuídas em três grupos experimentais: Grupo Combinação (COM, $n=8$ ), que recebeu $100 \mathrm{~mL}$ da combinação de butafosfan+cianocobalamina; Grupo Butafosfan (BUT, $n=8)$, que recebeu $100 \mathrm{~mL}$ de solução aquosa de Butafosfan; e Grupo Controle (CTL, $\mathrm{n}=7$ ), que recebeu $100 \mathrm{~mL}$ de solução fisiológica $0,9 \%$. Todos os animais recebram 5 doses subcutâneas de $20 \mathrm{~mL}$ a cada 24 horas, iniciando logo após o parto. Amostras de sangue foram coletadas nos dias $0,1,2,3,4,5,10,15,20$ e 28 para análises de cálcio, fósforo, ácidos graxos não esterificados (AGNEs) e $\beta$-hidroxibutirato (BHBA). Albumina, bilirrubina, colesterol total, haptoglobina e paraoxonase foram analisadas nos dias $0,3,5$, $10,15,20$ e 28. Foi avaliado o escore de condição corporal e a produção leiteira até 60 dias pós-parto. Níveis reduzidos de BHBA $(P<0,05)$ foram encontrados no grupo BUT. Animais com alto IFH apresentaram menores níveis de BHBA $(P<0,05)$ e tendência de redução nos níveis de AGNEs $(P<0,10)$, porém o IFH não foi influenciado pelos tratamentos. Apesar de o butafosfan reduzir os níveis de BHBA, o índice de funcionalidade hepática de vacas primímaras não foi influenciado pelos tratamentos.
\end{abstract}

Palavras chave: Fígado, fósforo orgânico, inflamação, vitamina B12.

\begin{abstract}
The objective of study was to evaluate the effects of butafosfan and cyanocobalamin on markers of energetic, mineral and inflammatory metabolism, milk production and the liver functionality index (LFI) of primiparous dairy cows. Twenty-three Holstein cows were allocated into: Combination group (COM, $n=8)$, receiving $100 \mathrm{~mL}$ of Catosal ${ }^{\circledR}$; Butafosfan group $(B U T, n=8)$ that received $100 \mathrm{~mL}$ of aqueous Butafosfan; and Control (CTL, $n=7)$, receiving $100 \mathrm{~mL}$ of $0.9 \%$ saline. All the animals received 100 $\mathrm{mL}$ ( 5 doses of $20 \mathrm{~mL}$ every $24 \mathrm{~h}$ ), subcutaneously, beginning soon after calving. Blood samples were collected on days $0,1,2,3$, $4,5,10,15,20$ and 28 for analysis of calcium, phosphorus, non-esterified fatty acids (NEFA) and $\beta$-hydroxybutyrate (BHB). The analysis of albumin, bilirubin, cholesterol, haptoglobin and paraoxonase were performed on days $0,3,5,10,15,20$ and 28 . We evaluated body condition score (BCS) and milk production ( $\mathrm{kg} /$ day) until day 60 after calving. The LFI was determined considering the plasma levels of total cholesterol, albumin and bilirubin on days 3 and 28 after calving. The BUT group showed lower BHB levels $(P<0.05)$ than CTL group. The animals from group with high LFI had lower concentrations of BHB $(P<0.05)$ and a trend of lower concentrations of NEFA $(P<0.10)$, whereas the animals with poor liver function (low) showed tendency to have lower level of albumin $(P<0.10)$. Howerver, the LFI was not affected by treatments $(P>0.05)$. In conclusion, despite the butafosfan and cyanocobalamin modulate energy metabolism in this study the hepatic functionality index was not affected by treatments.
\end{abstract}

Keywords: Inflammation, liver, organic phosphorus, vitamin B12.

\section{Introdução}

O período de transição de vacas leiteiras é marcado por mudanças endócrinas que causam alterações no metabolismo, predispondo os animais a diversos transtornos metabólicos (Drackley et al. 2001; Chapinal et al. 2011). A adaptação metabólica está ligada à capacidade hepática dos animais se adequarem às demandas homeorréticas da lactogênese, pois o fígado precisa atender ao alto requerimento de glicose e metabolizar o excesso de ácidos graxos não esterificados (AGNEs) (Drackley et al. 2001). Nestas condições de sobrecarga hepática, o fígado modifica seu padrão normal de síntese, tornando-se menos eficiente metabolicamente (Bobe et al. 2004), por isso alternativas que melhorem a funcionalidade hepática poderiam garantir uma melhor perfomance do fígado para enfrentar os desafios do pós-parto.

${ }^{*}$ Recebido em 5 de junho de 2016 e aceito em 7 de novembro de 2018.

**Universidade Federal de Pelotas (UFPel), Faculdade de Véterinária, Núcleo de Pesquisa, Ensino e Extensão em Pecuária (NUPEEC - www.ufpel.edu. br/nupeec), Pelotas, Rio Grande do Sul, Brasil.

Autor para correspondência: rubens_ap@yahoo.com.br 
O índice de funcionalidade hepática (IFH) permite uma avaliação do organismo da vaca ao final do primeiro mês de lactação (Bertoni \& Trevisi 2013; Zhou et al., 2016 e 2017) e tem sido utilizado para caracterizar a gravidade do estado inflamatório que os animais enfrentam nesse período. Sua determinação considera níveis séricos de três metabólitos hepáticos, albumina, colesterol e bilirrubina. Vacas com maior IFH possuem níveis reduzidos de AGNEs, corpos cetônicos e melhor desempenho produtivo (Bertoni et al. 2008, Trevisi et al. 2013, Montagner et at. 2016).

O butafosfan e a cianocobalamina são substãncias com efeito comprovado na redução dos níveis de AGNEs e betahidroxibutirato (BHBA), aumento da ingestão de matéria seca (IMS) e da produção leiteira (Pereira et al. 2013a,b). O butafosfan é uma fonte orgânica de fósforo, importante para o metabolismo energético celular (Berg et al. 2006). A cianocobalamina é um cofator enzimático fundamental para a gliconeogênese (Girard \& Matte 2005), mas em níveis reduzidos contribui para a lipidose hepática, pois reduz a taxa de utilização do propionato para a gliconeogêse (Girard \& Matte 2005). Como os níveis de fósforo (Grünberg et al. 2009) e cianocobalamina (Girard \& Matte 2005) estão reduzidos no pós-parto, o fornecimento dessas substâncias pode melhorar a condição energética da vaca leiteira.

Como nenhum estudo até o momento avaliou a utilização de estratégias farmacêuticas para melhorar o IFH, o objetivo deste estudo foi avaliar os efeitos do butafosfan e da cianocobalamina sobre a funcionalidade hepática de vacas leiteiras primíparas. Nossa hipótese é que melhore o status energético dos animais, garantindo ao fígado melhores condições de enfrentar os desafios do pós-parto, minimizando as alterações de síntese hepática que ocorrem durante a sobrecarga do órgão.

\section{Materiais e métodos}

O Comitê de Ética em Experimentação Animal (CEEA) da Universidade Federal de Pelotas aprovou todos os procedimentos deste projeto (cód. 4983), o qual foi realizado em uma fazenda leiteira comercial localizada no sul do Brasil (32 ${ }^{\circ} 16^{\prime} \mathrm{S}, 52^{\circ} 32^{\prime}$ E) de outubro a dezembro de 2014 . O rebanho da fazenda era composto por 800 vacas da raça Holandês em lactação, com sistema de manejo contendo pastagens e suplementação com silagem e concentrado. As vacas eram ordenhadas duas vezes ao dia e a média de produção era $5.500 \mathrm{Kg} / \mathrm{leite} / \mathrm{lactação.}$

Após o parto (2,6 $\pm 2,6$ dias), 23 vacas primíparas foram aleatoriamente distribuídas em três grupos: Grupo Combinação (COM, $\mathrm{n}=8$ ), que recebeu 5 doses de $20 \mathrm{~mL}$ da combinação de butafosfan (10\%) e cianocobalamina $(0,005 \%)$, Catosal ${ }^{\circledR} \mathrm{B}_{12}$ (Bayer Saúde Animal, Brasil); Grupo Butafosfan (BUT, $n=8$ ) que recebeu 5 doses de $20 \mathrm{~mL}$ de solução aquosa de butafosfan $10 \%$ e Grupo Controle (CTL, $n=7$ ), que recebeu 5 doses de $20 \mathrm{~mL}$ de solução fisiológica $\mathrm{NaCl}$ 0,9\% (Equiplex ${ }^{\circledR}$, Brasil). Apartir do parto foram feitas 5 aplicações subcutâneas a cada 24 horas, antes da alimentação da manhã e após a coleta de sangue.

Amostras de sangue foram coletadas do complexo arteriovenoso caudal de todos os animais, nos dias $0,1,2,3,4,5$, $10,15,20$ e 28 . As amostras foram centrifugadas por $15 \mathrm{~min}$ a $3.000 \mathrm{~g} / \mathrm{min}$ e os soros foram divididos em dois frascos e armazenados à $-20^{\circ} \mathrm{C}$. Análises de cálcio, fósforo, AGNEs e
BHBA foram realizadas nos dias $0,1,2,3,4,5,10,15,20$ e 28 , enquanto que as concentrações de albumina, bilirrubina, colesterol total, haptoglobina e paraoxonase foram determinadas nos dias $0,3,5,10,15,20$ e 28. As análises de cálcio, fósforo, albumina, colesterol e bilirrubina foram realizadas com kits comerciais (Labtest ${ }^{\circledR}$, Brasil), através de ensaio colorimétrico em espectrofotômetro semi-automático (SP 22, Biospectro ${ }^{\circledR}$, Brasil). $A$ atividade da paraoxonase foi determinada com kit comercial (ZeptoMetrix Corporation ${ }^{\circledR}$, USA) por espectrofotometria cinética (T80 UV/VIS, PG Instruments, Inglaterra). Os níveis séricos de haptoglobina foram determinados com kit comercial (Sigma Aldrich, Life Science ${ }^{\circledR}$, USA) em leitor de microplacas (TP-Reader, Thermo Plate ${ }^{\circledR}$, Brasil). AGNEs foram analisados colorimetricamente com kit comercial (Wako NEFA-HR, Wako Chemicals $^{\circledR}$, USA), de acordo com a técnica descrita por Ballou et al. (2009) e o BHBA foi analisado com kit comercial (Ranbut, Randox Laboratories Limited ${ }^{\circledR}$, UK). Os coeficientes de variação de todas as análises foram inferiores a $10 \%$.

O escore de condição corporal foi avaliado nos dias 0, 15 e 30 do estudo, conforme escala de 5 pontos (Wildman et al. 1982). A produção leiteira $(\mathrm{Kg} / \mathrm{dia})$ foi registrada até o dia 60 após o parto com auxílio do software de gerenciamento leiteiro Alpro (Delaval ${ }^{\circledR}$, USA).

O IFH foi determinado a partir das concentrações plasmáticas de colesterol total, albumina e bilirrubina total (medida indireta das enzimas responsáveis pela depuração hepática) nos dias 3 e 28 após o parto, conforme metodologia adaptada de Trevisi et al. (2012). Este método envolve duas etapas: na primeira são calculados índices para os três metabólitos, considerando os dias 0 e 28 do estudo, conforme as fórmulas: para albumina e colesterol e para bilirrubina onde $\mathbf{V}$ corresponde ao valor (concentração) do metabólito no dia 0 ou 28, e os coeficientes representam o percentual do resultado final dos índices (etapa 1) que é atribuído às concentrações do dia 0 ou à diferença entre os dois momentos. Tanto os resultados do dia 0 quanto a diferença entre os dias 28 e 0 da albumina e colesterol representam $50 \%$ do resultado do índice. A concentração de bilirrubina no dia 0 representa $67 \%$ e a diferença entre os dias 0 e 28 os outros 33\%. Na segunda etapa os índices parciais são padronizados conforme as médias e desvios padrões de vacas "saudáveis" (Bertoni et al. 2008). O IFH pode variar de -12 a +5 e indica respectivamente alta e baixa resposta inflamatória. Valores acima de 0 são considerados satisfatórios (Bertoni \& Trevisi 2013).

$$
\begin{gathered}
I F H=\left[\left(\frac{\text { I albumina }-17,71}{1,08}\right)+\left(\frac{\text { I colesterol }-2,57}{0,43}\right)\right. \\
-\left(\frac{\text { I bilirrubina }-6,08}{2,17}\right)
\end{gathered}
$$

I = Índice

Num segundo momento do presente estudo, os grupos foram desconsiderados e os animais foram categorizados em alto IFH $(-5,8$ à +19) 4 ou baixo IFH $(-9,3$ à -8,3) e as variáveis bioquímicas e zootécnicas foram novamente analizadas estatisticamente.

Os resultados foram analisados pelo software SAS 9.0 (SAS Institute Inc ${ }^{\circledR}$., Cary, NC, USA, 2004). Dados de albumina, bilirrubina, colesterol, haptoglobina, paraoxonase, AGNEs, BHBA, ECC, cálcio, fósforo, IFH e produção de leite foram 
submetidos à análise de variância (ANOVA) e procedimento MIXED para avaliar efeitos de grupo, período e a interação entre eles. Distribuição de frequência entre os grupos alto e baixo IFH foi analisada pelo Fisher's Exact Test. Valores de P $\leq 0,05$ foram considerados significativos e $\mathrm{P} \leq 0,10$ foi considerado tendência.

\section{Resultados}

O IFH das vacas deste estudo variou de $-9,3$ à $+1,9$, mas não diferiu entre os tratamentos $(P>0,05)$. Os valores médios de IFH foram de $-6,3 \pm 0,7,-5,9 \pm 1,3$ e $-7,9 \pm 1,1$ para os tratamentos BUT, COM e CTL, respectivamente (Tabela 1). Quando os animais foram categorizados em alto e baixo IFH, $87,5 \%(14 / 16)$ dos animais tratados com BUT ou COM tinham alto IFH ( $-5,8$ à $+1,9$, $n=14$, média $=-3,7 \pm 1,4)$, enquanto $57,14 \%(4 / 7)$ do CTL tinham baixo IFH $(-9,3$ à $-8,3, n=7$, média $=-8,9 \pm 0,1)$.

As concentrações de albumina, bilirrubina e colesterol, usadas para o cálculo do IFH, não diferiram entre os tratamentos $(P>0,05$, Tabela 1), mas a albumina teve uma tendência de redução nas vacas com baixo IFH $(P=0,06)$. Os níveis séricos de BHBA do tratamento BUT foram menores que do CTL $(P=0,04$, Tabela 1) e as concentrações de AGNEs não diferiam entre os tratamentos ( $P>0,05$, Tabela 1). Quando todos os animais foram agrupados e categorizados em alto e baixo IFH (independente dos tratamentos), as vacas com alto IFH tiveram menores níveis de $\operatorname{BHBA}(P=0,04)$ e tendência de redução nos níveis de $A G N E s$ $(P=0,07)$ do que vacas com baixo IFH.

Os níveis séricos de paraoxonase e haptoglobina não diferiram entre os tratamentos ( $P>0,05$, Tabela 1$)$, nem entre as categorias (alto ou baixo IFH). As concentrações séricas de cálcio e fósforo não foram influenciadas pelos tratamentos ( $P>0,05$, Tabela 1), nem diferiam entre as categorias (alto ou baixo IFH). A produção de leite e o ECC também não foram diferentes em nenhum momento $(P>0,05$, Tabela 1$)$.

\section{Discussão}

Falar aqui que já é sabido dos desafios do pós-parto...

Diversos trabalhos já avaliaram os efeitos do butafosfan e da cianocobalamina sobre marcadores do metabolismo energético e produção em vacas leiteiras (Fürll et al. 2010, Pereira et al. 2013a, b). Entretanto, esse é o primeiro estudo que avalia a influência do butafosfan e da cianocobalamina sobre o índice de funcionalidade hepática (IFH) de vacas leiteiras primíparas após o parto.

A segurança do uso da associação de butafosfan e cianocobalamina já foi demonstrada, provando que estas substâncias não sobrecarregam nem causam lesão hepática. Pelo contrário, permitem uma melhora na condição geral do órgão, através da redução da atividade beta-oxidativa dos AGNEs oriundos da mobilização lipídica (Kreipe et al. 2011), que é uma ocorrência comum e uma das principais preocupações metabólicas para vacas leiteiras no pós-parto recente.

Alguns trabalhos suportam nossa hipótese de que vacas em melhores condições energéticas possuem função hepática melhorada. Por exemplo, Grünberg et al. (2005) encontraram menores níveis séricos de fósforo inorgânico em vacas com melhor função hepática. Já Bertoni et al. (2008) e Trevisi et al. (2013) observaram que vacas com melhor função hepática possuiam baixos níveis séricos de AGNEs e corpos cetônicos. Além disso, Montagner et al. (2016) sugerem que o IFH pode servir como um importante biomarcador da síntese de proteínas de fase aguda e do intervalo até a primeira ovulação, podendo ser usado para definição de estratégias de melhora do desempenho produtivo e reprodutivo de vacas leiteiras.

No presente estudo, quando os animais foram categorizados em alto e baixo IFH, observamos que $85,7 \%$ das vacas tratadas com BUT ou COM tinham alto IFH, além de apresentarem

Tabela 1: Médias ( \pm erro padrão da média) de parâmetros metabólicos (paraoxonase, haptoglobina, albumina, colesterol, bilirrubina, cálcio, fósforo), IFH, ECC e produção leiteira de vacas primíparas tratadas com Butafosfan, Butafosfan e Cianocobalamina (Combinação) ou solução fisiológica (Controle)

\begin{tabular}{|c|c|c|c|c|c|}
\hline \multirow{2}{*}{ Parâmetro } & \multicolumn{3}{|c|}{ Tratamentos } & \multicolumn{2}{|c|}{ Valor de $\mathbf{P}$} \\
\hline & BUT & COM & CTL & Tratamento & Trat $\times$ Período \\
\hline $\mathrm{IFH}^{1}$ & $-6,38$ & $-5,94$ & $-7,97$ & 0,31 & \\
\hline $\mathrm{BHBA}^{2}(\mathrm{mmol} / \mathrm{L})$ & $0,421^{\mathrm{a}} \pm 0,02$ & $0,455^{\mathrm{ab}} \pm 0,02$ & $0,500^{\mathrm{b}} \pm 0,02$ & 0,04 & 0,88 \\
\hline $\mathrm{AGNEs}^{3}(\mathrm{mmol} / \mathrm{L})$ & $0,307 \pm 0,02$ & $0,359 \pm 0,02$ & $0,342 \pm 0,02$ & 0,10 & 0,12 \\
\hline Paraoxonase (U/L) & $91,9 \pm 5,4$ & $100,0 \pm 5,7$ & $106,2 \pm 5,8$ & 0,20 & 0,61 \\
\hline Haptoglobina (g/L) & $0,420 \pm 0,1$ & $0,500 \pm 0,1$ & $0,494 \pm 0,1$ & 0,77 & 0,43 \\
\hline Albumina (g/L) & $2,47 \pm 0,1$ & $2,59 \pm 0,1$ & $2,61 \pm 0,1$ & 0,23 & 0,85 \\
\hline Colesterol (mg/dL) & $103,1 \pm 3,6$ & $108,7 \pm 4,0$ & $109,5 \pm 4,1$ & 0,41 & 0,88 \\
\hline Bilirrubina (mmol/L) & $11,10 \pm 0,4$ & $11,57 \pm 0,5$ & $11,33 \pm 0,5$ & 0,83 & 0,07 \\
\hline Cálcio (mg/dL) & $9,62 \pm 0,1$ & $9,56 \pm 0,1$ & $9,29 \pm 0,1$ & 0,31 & 0,53 \\
\hline Fósforo (mg/dL) & $6,13 \pm 0,2$ & $6,05 \pm 0,2$ & $6,28 \pm 0,2$ & 0,76 & 0,45 \\
\hline $\mathrm{ECC}^{4}$ & $2,56 \pm 0,1$ & $2,58 \pm 0,1$ & $2,47 \pm 0,1$ & 0,49 & 0,77 \\
\hline Produção leiteira (L/dia) & $19,23 \pm 1,3$ & $20,13 \pm 1,2$ & $19,18 \pm 1,3$ & 0,84 & 0,47 \\
\hline
\end{tabular}

$a, b$ Letras diferentes na mesma linha indicam diferença estatística $(P<0,05)$. $P<0,10$ indicam tendência.

1 Índice de funcionalidade hepática: ${ }^{2}$ Beta hidroxibutirato; ${ }^{3}$ Ácidos graxos não esterificados; ${ }^{4}$ Escore de condição corporal. 
concentração reduzida de BHBA e tendência de menor AGNE, indicando efeito potencial do butafosfan e/ou cianocobalamina na melhora da função hepática através da otimização do metabolismo energético.

Quando em excesso, a mobilização lipídica pode favorecer o acúmulo de triacilgliceróis (TAG) no fígado e prejudicar a função hepática (Bobe et al. 2004). Todavia, em nosso estudo o IFH não diferiu entre os grupos (BUT, COM e CTL), apesar dos animais do grupo BUT estarem com menores níveis de BHBA (Tabela 1). Estes resultados são suportados pelo papel do fósforo no metabolismo hepático de carboidratos, já que muitos intermediários na rota gliconeogênica devem ser fosforilados (Berg et al. 2006; Hill et al., 2008), favorecendo também a metabolização dos AGNEs e reduzindo a formação de corpos cetônicos como o BHBA (Pereira et al. 2013a,b). Além disso, pequenas reduções nos níveis de fósforo citosólico após o parto podem afetar a atividade metabólica do fígado e comprometer a metabolização intensa de AGNEs (Grünberg et al. 2009), pois isso a administração de butafosfan, isolado ou combinado, pode trazer uma contribuição significativa para o metabolismo hepático nesta fase de alta demanda metabólica.

A maioria dos efeitos pronunciados do butafosfan e da cianocobalamina são encontrados em vacas multíparas, as quais geralmente possuem alta produção leiteira e, consequentemtente, um maior desafio metabólico e um balanço energético negativo mais severo (Fürll et al. 2010, Kreipe et al. 2011, Pereira et al. 2013b). Além disso, baixas concentrações de cianocobalamina e fósforo são comumente encontradas em vacas leiteiras de alta produção (Girard \& Matte 2005, Grünberg et al. 2009).

De acordo com os resultados de AGNEs, BHBA, produção de leite e ECC (Tabela 1), os animais deste estudo tiveram um baixo desafio metabólico, talvez por serem vacas primíparas sem alta produção leiteira (Kreipe et al. 2011, McAart et al. 2013). Além disso, situações estressantes como a troca de dieta, troca de ambiente, interação social, podem estimular respostas inflamatórias que afetam a funcionalidade hepática. E ainda, a transição para a lactação em vacas primíparas pode ser bem mais estressante que em vacas multíparas por ser a primeira vez que isso ocorre, fazendo com que os animais tenham processos inflamatórios subclínicos mesmo antes do parto (Bertoni et al. 2008). No presente estudo, todos os animais apresentaram IFH baixos (Tabela 1), possivelmente por terem apresentado respostas inflamatórias em virtude dos desafios metabólicos do periparto.

As concentrações sanguíneas de albumina dos animais do presente estudo estavam abaixo dos padrões fisiológicos para a espécie (2,7-3,8 mg/dL, Tabela 1$)$, o que pode indicar a incapacidade hepática para sua síntese, já que situações estressantes do periparto podem causar respostas inflamatórias subclínicas, comprometendo a função do órgão (Bertoni et al. 2008). Por outro lado, os níveis de bilirrubina estavam acima dos padrões fisiológicos para vacas "saudáveis" no pós-parto, indicando a incapacidade das enzimas hepáticas fazerem sua depuração, provavelmente pela baixa funcionalidade do órgão (Bertoni \& Trevisi 2013).

Apesar das proteínas de fase aguda (haptoglobina e paraoxonase) não serem utilizadas para o cálculo do IFH, elas são importantes indicadores de processos inflamatórios e estão associadas às mudanças no padrão de síntese hepática (Bertoni et al. 2008). Neste estudo, a haptoglobina alcançou níveis normais (abaixo de 0,2 g/L, Bertoni et al. 2008) apenas no final do primeiro mês de lactação. Em geral, os maiores níves de haptoglobina $(0,4$ a $0,5 \mathrm{~g} / \mathrm{L})$ são encontrados na primeira semana após o parto (Bertoni \& Trevisi 2013; Schneider et al., 2013). Todavia, em nosso estudo os níveis mais elevados foram encontrados no dia do parto, o que pode estar relacionado a possíveis processos inflamatórios subclínicos ainda no pré-parto, relacionados a situações estressantes do período de transição para a lactação (Bertoni et al. 2008).

Poucos estudos avaliaram a ação direta do butafosfan e da cianoconalamina sobre marcadores inflamatórios de vacas leiteiras. Por exemplo, Nuntaprasert \& Watanapongchart (2006) observaram redução da resposta inflamatória de fase aguda e diminuição dos níveis séricos de haptoglobina, fator de necrose tumoral e interleucina-6, em animais tratados com butafosfan e cianocolbalamina e avaliados alguns minutos depois. Todavia em nosso estudo as avaliações foram feitas um dia após o tratamento, o que pode ter limitado a detecção das respostas positivas, que são rápidas, considerando a meia vida plasmática do butafosfan de apenas 116 minutos (EMEA, 2000).

Alguns trabalhos já comprovaram os efeitos do butafosfan e da cianocobalamina sobre os marcadores do metabolismo energético, principalmente em vacas leiteiras mutíparas, que em geral possuem maior produção leiteira e maior desafio metábolico (Deniz et al. 2008, Fürll et al. 2010, Pereira et al. 2013b). Entretanto, como as vacas primíparas do presente estudo não apresentavam alta produção leiteira e o desafio metabólico após o parto não era tão intenso, os efeitos das estratégias testadas sobre a funcionalidade hepática não foram tão pronunciados. Possivelmente melhores resultados possam ser alcançados se as estratégias forem utilizadas antes do parto de vacas primíparas, momento em que possivelmente iniciamse os processos inflamatórios subclínicos do periparto; ou no pós-parto de vacas multíparas, momento em que alta produção aumenta o desafio metabólico para atender suas demandas energéticas.

\section{Conclusão}

A utilização do butafosfan e/ou cianocobalamina não alterou o índice de funcionalidade hepática em vacas leiteiras de primeira lactação. Outros estudos são necessários para avaliar o uso destes compostos no pré-parto de primíparas ou no pós-parto de vacas multíparas de alta produção e elevado desafio metabólico.

\section{Agradecimentos}

Aos integrantes do Núcleo de Pesquisa, Ensino e Extensão em Pecuária (NUPEEC) pelo auxílio na realização do projeto, à Granjas 4 Irmãos S.A. pela disponibilidade de infra-estrutura para o estudo e à Capes e CNPq pelo apoio financeiro para a realização do projeto. 


\section{Referências}

BALLOU, M.A.; Gomes, R.C.; Juchem, S.O.; DePeters, E.J. Effect of dietary supplemental fish oil during the peripartum period on bloodmetabolites and hepatic fatty acid compositions and total triacylglycerol concentration of multiparous Holstein cows. Journal of Dairy Science 92, 657-669, 2009.

BERG, J.M.; Tymoczo, J.L.; Stryer, L. Glycolysis and gluconeogenesis. In: Biochemistry, Sixth Ed. W. H. Freeman and Company, New York, NY, USA, pp. 433-474, 2006.

BERTONI, G.; Trevisi, E. Use of the liver activity index and other metabolic variables in the assessment of metabolic health in dairy cows. Veterinary Clinics of North America Food Animal Practice 29, 413-431, 2013.

BERTONI, G.; Trevisi, E.; Bionaz, M. Effects of inflammatory conditions on liver activity in puerperium period and consequences for performance in dairy cows. Journal of Dairy Science 91, 3300-3310, 2008.

BOBE, G.; Young, J.W.; Beitz, D.C. Pathology, etiology, prevention, and treatment of fatty liver in dairy cows. Journal of Dairy Science 87, 3105-3124, 2004.

CHAPINAL, N.; Carson, M.E.; Duffield, T.F.; Capel, M.; Godden, S.; Overton, M.; Santos, J.E.P.; Leblanc, S.J. The association of serum metabolites with clinical disease during the transition period. Journal of Dairy Science 94, 489-4903, 2011.

DENIZ, A; Westphal, B; Illing, C. Effects of prepartum metaphylactic treatment with Catosal on postpartum metabolic functions in cows. Oral and Poster Presentations. Proceedings of the $\mathrm{XXV}^{\text {th }}$ World Buiatrics Congress. Budapest, Hungary, $\mathrm{pp}$. 26-31, 2008.

DRACKLEY, J.K.; Overton, T.R.; Dowlen, H.H. Adaptations of glucose and long-chain fatty acid metabolism in liver of dairy cows during the periparturient period. Journal of Dairy Science 84, E110-E112, 2001.

EMEA. The European Agency for the Evaluation of Medicinal Products. Veterinary Medicines and Information Technology Unit. EMEA/MRL/734/00-FINAL, p 1-2. EMEA, London, UK, 2000.

FÜRLL, M.; Deniz, A.; Westphal, B.; Illing, C., Constable, P.D. Effects of multiple intravenous injections of butaphosphan and cyanocobalamin on the metabolism of periparturient dairy cows. Journal of Dairy Science 93, 4155-4164, 2010.

GIRARD, C.L and Matte, J.J. Effects of intramuscular injections of vitamin B12 on lactation performance of dairy cows fed dietary supplements of folic acid and rumen-protected methionine. Journal of Dairy Science 88, 671-676, 2005.

GRÜNBERG, W.; Constable, P.D.; Schröder, U.; Staufenbiel, R.; Morin, D.E.; Rohn, M. Phosphorus homeostasis in dairy cows with abomasal displacement or abomasal volvulus. Journal of Veterinary Internal Medicine 20, 1471-1478, 2005.

GRÜNBERG, W.; Staufenbiel, R.; Constable, P.D.; Dann, H.M.; Morin, D.E.; Drackley, J.K. Liver phosphorus content in HolsteinFriesian cows during the transition period. Journal of Dairy Science 92, 2106-2117, 2009.

HILL, S.R.; Knolton, K.F.; Kebreab, E.; France, J.; Hanigan, M.D. A model of phosphorus digestion and metabolism in the lactating dairy cow. Journal of Dairy Science 91, 2021-2032, 2008.
KREIPE, L.; Deniz, A.; Bruckmaier, R.M.; van Dorland, H.A. First report about the mode of action of combined butaphosphan and cyanocobalamin on hepatic metabolism in nonketotic early lactating cows. Journal of Dairy Science 94, 4904-4914, 2011.

MCART, J.A.A.; Nydam, D.V.; Oetzel, G.R.; Overton, T.R.; Ospina, P.A. Review - Elevated non-esterified fatty acids and ß-hydroxybutyrate and their association with transition dairy cow performance. The Veterinary Journal 192, 560-570, 2013.

MONTAGNER, P.; Krause, A.R.T.; Schwegler, E.; Weschenfelder, M.M.; Rabassa, V.R.; Schneider, A.; Pereira, R.A.; Brauner, C.C.; Del Pino, F.A.B.; Gonçalves, F.M.; Corrêa, M.N. Reduction of liver function delays resumption of postpartum ovarian activity and alters the synthesis of acute phase proteins in dairy cows. Research in Veterinary Science, v. 106, p. 84-88, 2016.

NUNTAPRASERT, A.; Watanapongchart, S. A field effect of Catosal on the acute phase response, performance and metabolic disorders of farrowing sows in Thailand. In: Proceedings of the 19th International Pig Veterinary Society Congress, Copenhagen, Denmark, pp. 487, 2006.

PEREIRA, R.A.; Fensterseifer, S.; Barcelos, V.B.; Martins, C.F.; Schneider, A.; Schmitt, E.; Pfeifer, L.F.M.; Delpino, F.A.B.; Corrêa, M.N. Metabolic parameters and dry matter intake of ewes treated with butaphosphan and cyanocobalamin in the early postpartum period. Small Ruminant Research 114, 140-145, 2013a.

PEREIRA, R.A.; Silveira, P.A.S.; Montagner, P.; Schneider, A.; Schmitt, E.; Rabassa, V.R.; Pfeifer, L.F.M.; Delpino, F.A.B.; Pulga, M.E.; Corrêa, M.N. Effect of butaphosphan and cyanocobalamin on postpartum metabolism and milk production in dairy cows. Animal 7, 1143-1147, 2013b.

SCHNEIDER, A.; Corrêa, M.N.; Butler, W.R. Acute phase proteins in Holstein

cows diagnosed with uterine infection. Research in Veterinary Science 95, 269-271, 2013.

TREVISI, E.; Amadori, M.; Cogrossi, S.; Razzioli, E.; Bertoni, $\mathrm{G}$. Metabolic stress and inflammatory response in high-yielding, periparturient dairy cows. Research in Veterinary Science 93, 695-704, 2012.

TREVISI, E.; Ferrari, A.; Piccioli-Cappeli, F.; Grossi, P.; Bertoni, G. An additional study on the relationship between the inflammatory condition at calving time and net energy efficienty in dairy cows. EAAP Scientific Series. In: Crovetto, G. M. (Ed). Energy and protein metabolism and nutrition, v. 127. Wageningen Academic Publishers, Wageningen, Netherlands, pp. 489-490, 2010.

WILDMAN, O.E.E.; Jones, G.M.; Wagner, P.E. A dairy cow body condition scoring system and its relationship to selected production characteristics. Journal of Dairy Science, v.65, n.3, p.495-501, 1982.

ZHOU, Z.; Loor, J.J.; Piccioli-Cappelli, F.; Librandi, F.; Lobley, G.E.; Trevisi, E. Circulating amino acids in blood plasma during the peripartal period in dairy cows with different liver functionality index. Journal of Dairy Science 99, 2257-2267, 2016.

ZHOU, Z.; Trevisi, E.; Luchini, D.N.; Loor, J.J. Differences in liver functionality indexes in peripartal dairy cows fed rumen-protected methionine or choline are associated with performance, oxidative stress status, and plasma amino acid profiles. Journal of Dairy Science 100, 6720-6732, 2017. 